\title{
EFFECT OF TISSUE CONDITIONER COMBINED WITH NYSTATIN ON GROWTH OF CANDIDA ALBICANS IN COMPLETE DENTURE WEARERS
}

\author{
Eman Mostafa Ahmed Ibraheem* and Wessam M. Dehis*
}

\begin{abstract}
Aim: this study aimed to evaluate the effect of incorporation of Nystatin antifungal with tissue conditioner applied to the maxillary complete denture on growth of Candida Albicans.

Materials and methods: Fifteen completely edentulous patients were selected and conventional complete dentures were fabricated for each patient. Viscogel tissue conditioner was applied at denture delivery. Isolation and identification of Candida albicans were done using Agar core inhibition assay after two months from wearing the denture. Then, a new application of viscogel mixed with Nystatin antifungal was applied replacing the first one and after additional two months from wearing the denture, Isolation and identification of Candida albicans was done.
\end{abstract}

Results: there was no significant difference was observed before and after application of Nystatin antifungal.

Conclusion: tissue conditioner when mixed with Nystatin antifungal showed no marked effect on inhibition of Candida Albicans.

KEY WORDS: Tissue conditioner, Nystatin, antifungal, Candida Albicans.

\section{INTRODUCTION}

Complete denture prosthesis involves the replacement of the lost natural teeth and the associated structures of the maxilla and the mandible. One of the goals that the dentist has to achieve is to produce a denture that is retentive and stable in place which in turn enhances both function and esthetics. ${ }^{(1)}$
Candida Albicans is a component of the normal oral flora, but local and systemic factors can transform this commensal C. Albicans to a pathogen causing Denture Stomatitis. ${ }^{(2)}$

Denture Stomatitis is multifactorial in origin. Denture Stomatitis is an inflammatory condition involving the tissues covering the denture base. It is more common in maxilla and rare in mandible. ${ }^{(3)}$

* Researcher in Fixed and Removable Prosthodontic Department, National Research Centre, Cairo, Egypt 
Candida Albicans infection and trauma are significant causes for Denture Stomatitis. Predisposing factors include poor denture hygiene, bad denture wearing habits, xerostomia, medications and nutritional deficiencies. ${ }^{(4)}$

This condition can be managed by improvement of denture hygiene, use of tissue conditioner to improve denture adaptation, and topical application of antifungal when presence of yeast has been confirmed. ${ }^{(5)}$

Tissue conditioner improves the adaptation between the denture and denture bearing tissues and thus allow recovery of the tissues. ${ }^{(6)}$

Tissue conditioner promotes or supports in vivo candida colonization in short term use of tissue conditioner. However, in vitro use of tissue conditioner for long periods of time, intensify the formation of fungal biofilms. Presence of saliva and serum pellicle promotes colonization of antifungal by Candida. ${ }^{(7)}$

The first step of colonization is fungal adhesion to the surface of material then, fungi can penetrate into the material. ${ }^{(8)}$

Combination of tissue conditioner and antifungal agent provides prolonged action of the drug, low cost and helps rapid tissue recovery after trauma. ${ }^{(9,10)}$

\section{AIM OF THE STUDY}

In this study we compared the effect of tissue conditioner before and after incorporation of Nystatin antifungal on the growth of Candida albicans.

\section{MATERIALS AND METHODS}

Fifteen male completely edentulous patients were selected from the out-patient clinic, Fixed and Removable Prosthodontic Department, National Research Centre, Cairo, Egypt. Age range was between 45 and 65 years. Patients were non- smokers and having healthy mucosal coverage free from any signs of inflammation, ulceration or hyperplasia. All patients had well-formed residual alveolar ridge free from severe bilateral undercuts and bony spicules or sharp ridges. All patients were free from any T.M.J. disorders and having angle class1 ridge relationship. Patients also, were Free from any systemic diseases that may affect the oral condition e.g. diabetes mellitus and anaemia and had no previous denture experience.

Patients were evaluated before inclusion in this study through history and clinical evaluation Each patient received upper and lower complete dentures that were constructed in a conventional manner.

At the visit of denture delivery, patients were motivated to practice oral hygiene measures as to remove the dentures overnight, to remove the dentures after every meal and rinse them under running water only and not to keep the dentures in any type of denture cleansers but keep it only in a cup of tap water.

Before application of tissue conditioner, patients were instructed to rinse with $0.2 \%$ chlorhexidine mouth wash once to remove any Candida attached in their mouth (which may affect the results) and swab was taken from the palate and cultured to ensure that no Candida exists. Then, viscogel tissue conditioner was mixed in sterile dippen dish according to manufacturer instructions and applied to the fitting surface of maxillary and mandibular dentures. The dentures were inserted with instruction to come back after two months.

\section{Isolation and identification of Candida albicans}

After two months, samples were obtained by Vigorous rubbing of all the fitting surface of maxillary complete denture using sterile cotton tipped wooden swab for each patient.

Each swab was inoculated immediately in sterile glass tube containing $2 \mathrm{ml}$ of Sabouraud's Dextrose Broth (10gm peptone, 20gm dextrose and $1000 \mathrm{ml}$ 
distilled water) and was transferred to the laboratory within one hour20 . $\mu$ l from each tube of Sabouraud's Dextrose Broth was plated on Sabouraud's Dextrose Agar plate to which chloramphenicol was added (40 gm dextrose, $10 \mathrm{gm}$ peptone, $15 \mathrm{gm}$ agar, and 1000 $\mathrm{ml}$ distilled water), and spreaded using a sterile bent glass rod.

The Sabouraud's Agar plates were incubated at $37 \mathrm{oC}$ for 48 hours, after which all the resulting yeast colonies were counted and identified as Candida albicans by germ tube test. Fig. (1)

In the germ tube test, yeast colony was suspended in $0.5 \mathrm{ml}$ of human serum. The mixture was incubated at $37^{\circ} \mathrm{C}$ for 2 hours, and then a drop of the suspension was examined under the light microscope for germ tubes. Fig. (2)

If no colonies were observed after 2 days of such incubation, negative results were reported. The dentures were mechanically cleaned from Candida using a soft toothbrush, and another swab was taken and cultured as previous to ensure that no Candida exists on the dentures.

Again, The patients were instructed to rinse with $0.2 \%$ chlorhexidine mouth wash once to remove any Candida attached in their mouth as a result of wearing the dentures and swab was taken from the palate and cultured as previous to ensure that no Candida exists.

The first application of viscogel was replaced by new application of tissue conditioner mixed with Nystatin antifungal (5\%wt/wt TC, equivalent to $100,000 \mathrm{IU})$ with the powder of tissue conditioner and the dentures were reinserted and patients were again motivated for proper oral hygiene measures and instructed to come back after another two months for sample collection as were described before.

Samples were treated as previously and the data were collected and tabulated for statistical analysis.

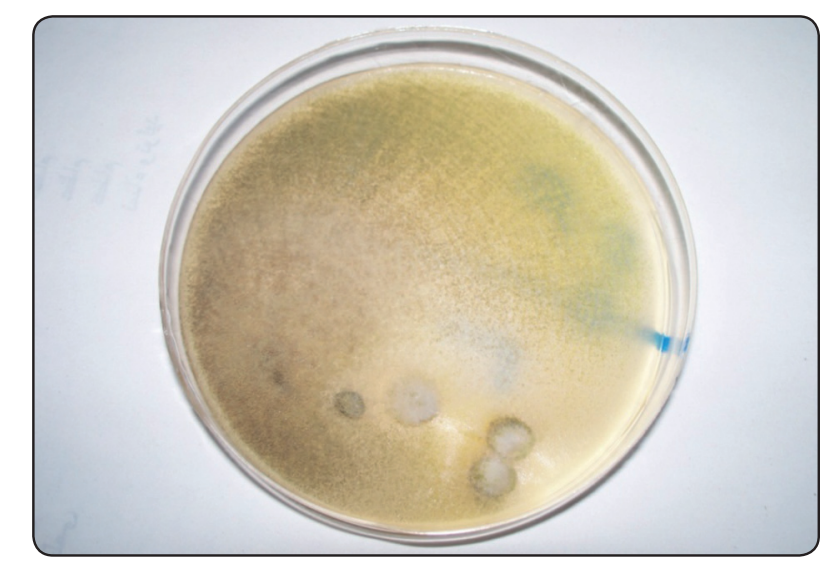

Fig. (1) positive candidal appearance.

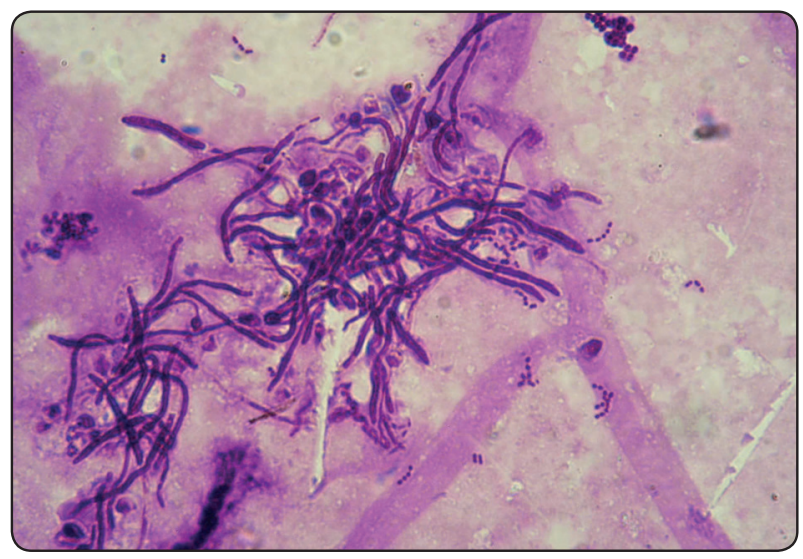

Fig. (2) Germ tubes

\section{RESULTS}

Data were presented as mean and standard deviation (SD) values. A logarithmic transformation ( $\log 10$ transformation) of each colony forming unit (CFU) count was performed to normalize the data before statistical evaluation because of the high range of counts.

The significance level was set at $\mathrm{P} \leq 0.05$. Statistical analysis was performed with SPSS 16.0 (Statistical Package for Scientific Studies) for Windows.

\section{Comparison including cases with Candida growth}

Paired t-test was used to compare between CFU before and after addition of Nystatin antifungal to the tissue conditioner applied to the fitting surface 
of the maxillary denture.

The mean and standard deviation values of $\log _{10}$ values of $\mathrm{CFU}$ were $3.07 \pm 0.88$ and $2.25 \pm 0.47$ before and after addition of Nystatin the tissue conditioner in the fitting surface of the denture.

There was non-statistically significant decrease in mean $\log _{10}$ values of CFU after addition of

Nystatin the tissue conditioner in the fitting surface of the denture $(\mathrm{P}$-value $=0.063)$.

TABLE (1) Showing comparison between $\log 10$ values of CFU before and after addition of Nystatin the tissue conditioner in the fitting surface of the denture

\begin{tabular}{ccrrrr}
\hline & \multicolumn{2}{c}{ After } & \multicolumn{2}{c}{ Before } \\
& \multicolumn{1}{c}{ SD } & Mean & SD & Mean \\
0.063 & 0.47 & 2.25 & 0.88 & 3.07
\end{tabular}

\section{Comparisons for cases with Candida albicans growth}

Paired t-test was used to compare between CFU before and after addition of Nystatin to tissue conditioner in the fitting surface of the denture.

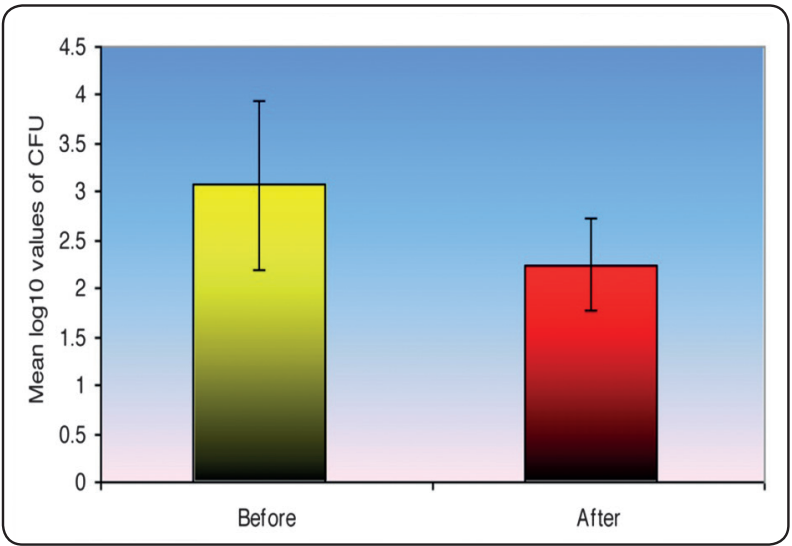

Fig. (3) Bar chart representing mean and standard deviation values of $\log _{10}$ values of CFU before and after addition of Nystatin the tissue conditioner in the fitting surface of the denture
The mean and standard deviation values of $\log _{10}$ values of CFU were $2.91 \pm 0.98$ and $2.33 \pm$ 0.51 before and after addition of Nystatin to tissue conditioner in the fitting surface of the denture.

There was non-statistically significant decrease in mean $\log _{10}$ values of CFU after addition of Nystatin $(\mathrm{P}$-value $=0.187)$.

TABLE (2) Showing the comparison between $\log 10$ values of $\mathrm{CFU}$ before and after addition of Nystatin to the fitting surface of the denture

\begin{tabular}{|c|c|c|c|c|}
\hline \multirow{2}{*}{$P$-value } & \multicolumn{2}{|c|}{ After } & \multicolumn{2}{c|}{ Before } \\
\cline { 2 - 5 } & SD & Mean & SD & Mean \\
\hline $\mathbf{0 . 1 8 7}$ & 0.51 & 2.33 & 0.98 & 2.91 \\
\hline
\end{tabular}

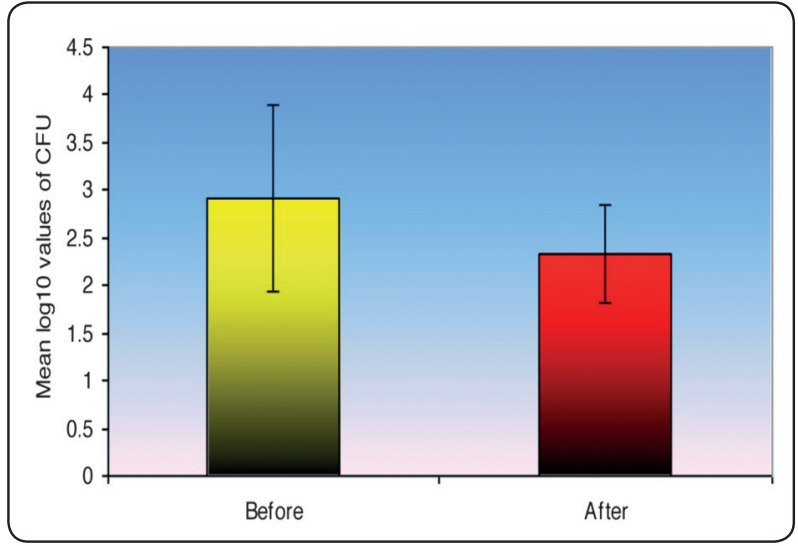

Fig. (4) Bar chart representing mean and standard deviation values of $\log _{10}$ values of CFU before and after addition of Nystatin the tissue conditioner in the fitting surface of the denture

\section{DISCUSSION}

Swabbing technique was employed in this study for isolation of the yeast Candida albicans as a simple and efficient method for oral yeast isolation..$^{(11)}$

The swabs were placed immediately in tubes of fresh Sabouroud Dextrose (SAB) Broth and 
transferred to the laboratory within one hour as the swabs should be sent to the laboratory in a standard transport medium. ${ }^{(12)}$

$20 \mathrm{ml}$ from each tube was plated on Sabouraud's Dextrose Agar plate as isolation is best made on agar plates as the high dextrose concentration and acidic $\mathrm{pH}$ of the formula permits selectivity of fungi. Chloramphenicol was added as antifungal to reduce bacterial contamination and the plates were incubated at $37^{\circ} \mathrm{C}$ for 48 hours. The resultant colonies were counted and identification of Candida albicans was carried out using germ tube test as the formation of germ tubes in serum is a characteristic of most clinical isolates of Candida albicans. ${ }^{(13,14)}$

This work was carried out on fifteen patients. The results showed that Candida was isolated from seven patients (45\% of patients) and Candida albicans was isolated from four patients $(25 \%$ of patients) before and after addition of Nystatin antifungal to the tissue conditioner applied in the fitting surfaces of the dentures. ${ }^{(15)}$

The presence of either Candida or Candida albicans in some individuals and its absence in others can be attributed to individual variation as the commensal existence of intraoral Candida species varies from $20 \%$ to $50 \%$ in a healthy dentulous population and up to $75 \%$ in a population wearing dentures, while the incidence of Candida albicans isolated from the oral cavity has been reported to be $30-40 \%$ in healthy adults and $50-65 \%$ in people with removable dentures. ${ }^{(16)}$

It was noticed that there is decrease in the number of colonies of either Candida or Candida albicans but this decrease was not statistically significant before and after addition of Nystatin. This could be attributed to the effect of saliva which reduces the adhesion of Candida albicans and thus diminishes the effect of Nystatin. This was in accordance with the finding that Candida adhesion to dentures could be influenced by saliva. Nystatin incorporated with tissue conditioner isn't stable inside the saliva and their concentration gradually reduced. ${ }^{(17)}$
This is in agreement with clinical studies ${ }^{(18)}$, where patients with low or impaired salivary flow and/or composition presented higher Candida species counts when compared with saliva from patients with normal salivary flow.

This influence might be regulated by specific interactions between the cellular adhesions and receptors in the salivary pellicle as well as by the action of salivary proteins as a source of nutrients for microbiological growth. However, these proteins might also act by blocking the locations of adhesion originally present on substrates. ${ }^{(19)}$

The effect of pooled un-stimulated saliva produced a statistically significant reduction in adhesion. This reduction in adhesion also diminished the effect that Nystatin had on the inhibition of candida growth. ${ }^{(20)}$

The decrease in the number of colonies of either Candida or Candida albicans after addition of Nystatin to the tissue conditioner applied in the fitting surface of the dentures could be due inhibition of formation of biofilm to which denture plaque will be formed and C. albicans under gores colonization. ${ }^{(21,22)}$

\section{REFRENCES}

1. Zarb et al: prosthodontics treatment of edentulous patients. Complete denture and implant supported prostheses. Elservier Mosby, thirteenth ed, 2013, 28.

2. Bokor-Bratic M, Cankovic M, Dragnic N. Unstimulated whole salivary flow rate and anxiolytics intake are independently associated with oral Candida infection in patients with oral lichen planus. Eur J Oral Sci 2013; 121(5):427-33.

3. Martinez RF, Jaimes-Aveldañez A, Hernández-Pérez F, Arenas R, Miguel GF. Oral Candida spp carriers: its prevalence in patients with type 2 diabetes mellitus. An Bras Dermatol $2013 ; 88(2): 222-5$

4. De Freitas EM, Nobre SA, Pires MB, Faria RV, Batista AU, Bonan PR. Oral Candida species in head and neck cancer patients treated by radiotherapy. Auris Nasus Larynx 2013;40(4):400-4 
.5 Nakahara T, Harada A, Yamada Y, Odashima Y, Nakamura $\mathrm{K}$, Inagaki $\mathrm{R}$, et al. Influence of a new denture cleaning technique based on photolysis of $\mathrm{H}(2) \mathrm{O}(2)$ the mechanical properties and color change of acrylic denture base resin. Dent Mater J 2013;32(4):529-36

6. Rathore P, Hegde A, Ginjupalli K, Upadhya N. Evaluation of antifungal activity of additives to resi-lient liners: an in vitro pilot study. Trends in Bioma-terials and Artificial Organs 2009; 23(1):6-9.

7. Greets G, Stuhlinger M and Basson N. Effect of antifungal denture liner on the saliva yeast count in patients with denture stomatitis. A pilot study. L Oral Rehab. 2008; 35: 664-69

8. Chladek $\mathrm{G}$ et al. antifungal activity of denture soft lining material modified by silver nanoparticles. A pilot study. Int J Mol Sci 2011; 12: 4735-44

9. Fallah-tafti A, et al. Stability and duration of antifungal effects of Nystatin and Fluconazole mixed with a tissue conditioner on colonization of Candida Albicans (in vitro). Original article. J Res Dent Sci. Spring 2014; 11(1): 20-25

10. Chopde $\mathrm{N}$ et al. in vitro antifungal activity of two tissue conditioners combined with nystatin, miconazole and fluconazole against candida albicans. J Cont Dent Pract. 2012, 13 (5), 695-698

.11 .Uchirmaru M, Sakali T, Morio R, Shiota S, Shibata Y, Deguch $\mathrm{m}$, et al.Antimicrobial and antifungal effects of tissue conditioners containing a Photocatalyst. Dent Mater J 2011;30(5):691-9

12. Radnai M, Whiley R, Friel T, Wright PS. Effect of antifungal gels incorporated into a tissue conditioning material on the growth of Candida albicans.Gerodontology 2010; 27(4):292-6.
13. Baveja C. "Medical mycology," in Text Book of Microbiology for Dental Students, pp. 322-323, Arya Publications, Delhi, India, 3rd edition, 2010.

14. Pfaller M, Houston A, and Coffmann S, "Application of CHROM agar Candida for rapid screening of clinical specimens for Candida albicans, Candida tropicalis, Candida krusei, and Candida (Torulopsis) glabrata," Journal of Clinical Microbiology, vol. 34, no. 1, pp. 58-61, 1996.

15. Cannon RD and Chaffin WL. Oral colonization by Candida albicans. Crit Rev Oral Biol Med. 1999; 10 (3):359-83.

16. Khozeimeh F. A comparative study of Candida albicans means colony counts and blood group antigens in the saliva of healthy subjects. Dent Res J (Isfahan). 2014 MarApr; 11(2): 240-243.

17. El-Charkawi H, el-Said EA, Safout HM, elRaghi N. Effect of addition antimicrobial agents to denture reliners. Egypt Dent J 1994; 40(3):785-90.

18. Silva M. Influence of artificial saliva in biofilm formation of Candida albicans in vitro. Braz. oral res. vol.26 no.1 São Paulo Jan./Feb. 2012

19. Jin Y et al. Biofilm formation of Candida albicans is variably affected by saliva and dietary sugars. Archives of Oral Biology (2004) 49, 789-79.

20. Moura $\mathbf{J}$ et al. Influence of acrylic resin polymerization methods and saliva on the adherence of four Candida species. J Prosthetic Dent . 96, (3), September 2006, 205-211

21. Thomas $\mathrm{C}$ and Nutt $\mathrm{G}$. the in vitro fungicidal properties of viscogel alone and combined with nystatin and amphotericin B. J Oral Rehabil, 1978, 5, 162-72

22. Gupta $\mathrm{H}$ et al. An Innovative Method of Incorporating Antifungal Agents into Tissue Conditioners: An In Vitro Study. Trends Biomater. Artif. Organs, 25(2), 63-66 (2011) 